\title{
MHC class II invariant chains in antigen processing and presentation
}

\author{
Norbert Koch, Joachim Lipp, Ulrich Pessara, Klaus \\ Schenck, Christopher Wraight and Bernhard Dobberstein
}

Most protein antigens cannot elicit a T-cell response unless they are processed to peptides, which are then presented to $T$ lymphocyes by surface $M H C$ class $I I$ molecules. Recent evidence suppors an essential role of the invarian chan associated with class $/ /$ MHC polypeptides in antigen processing.

The main function of the immune system is the natural defense against infections. Detalled research over recent years has illuminated the cellular and molecular nature of this immune reaction. Immune cells can respond to foreign antigens by generation of cytotoxic T lymphocytes or by antibody production from $B$ cells. These responses are tightly controlled by class $I$ and class 11 polypeptides that are encoded by genes of the major histo. compatibility complex (MHC). Class I proteins bind cellular peptides (e.g. virus antigens) and present them on the cell surface to cytotoxic T lymphocytes. These cytotoxic $T$ cells recognize the class 1 polypeptides and the peptide with their antigen receptor and sub. sequently lyse these cells ${ }^{1}$. Class II proteins present peptides mainly derived from extracellular antigens, to Thelper lymphocytes ${ }^{2}$ which subsequently give help to B lymphocytes to produce antibodies against the antigen. Before the antigen is presented to Tlymphocytes it must be processed ${ }^{3}$ (see also a recent review in $T I B S 13,27-29,1988$ )

In this report we will consider the processing and presentation of class II restricted antigens. Processing involves denaturation of antigen and its digestion to peptides. Not all peptides are presented, however. Selection of immunogenic peptides is mediated by binding of the peptide to one of the various class II molecules followed by recognition by $T$ lymphocytes ${ }^{4}$. Since class II proteins can present ${ }^{5}$ but not process antigens ${ }^{3}$, other cellular el-

N. Koch, U. Pessara, K. Schenck and C. Wraight are at he Institue for Immwnology and Genetic, German Cancer Research Center, D-6900 Heidel. berg. FRG. 2. Lipp and B. Dobberstein are at the European Molecular Blology Laboratory. Heidelberg, FRG. ements are requited for the intemaliz ation. transport and digestion of antigen and subsequent interaction of the peptides with class II polypeptides. Several reviews have been published over the last two years that discuss the cellular events in antigen processing 6.7 . Here we focus on the role of class 11 associated invariant chain in antigen processing and presentation.

\section{Internalization and processing of antigen}

Antigen processing by antigen presenting cells (APCS) requires internalization of antigen as its first step. Endocytosed material notmally ends up in lysosomes where the protein antigens are fully degraded to amino acids. Since antigen presentation requires peptides not smaller than 8 to 12 amino acids, an intermediate event that leads to partial degradation of protein antigens can be postulated. It is conceivable that this processing occurs in a compartment with limited proteolytic activity. Certain endosomes have been found to contain membrane-bound cathepsin $D$, hence this enzyme is a candidate for the partial degradation of antigen ${ }^{8}$. How could this step fit into the so far unknown mechanism by which external antigen appears as class II-bound processed peptide on the surface of the cell? The various stages of antigen processing can be defined thus:

(1) The binding of antigen to cell sur face structures of APCs, which might be a random event, occurring by various interactions due to the physicochemical properties of the antigen and appropriate cell surface molecules.

(2) The uptake of antigen by fuidphase or receptor-mediated endocytosis into early or late endosomes or a prelysosomal compartment.
(3) The processing of antigen by as yet undefined enzymes.

(4) The interception of processed antigen with class II molecules in the processing compartment.

Structural features of class II polypeptides and invariant chains

Class 11 proteins consist of two integral polymorphic membrane proteins ( $\alpha$ and $\beta$-chains) that expose their $C$ terminus on the cytoplasmic side (Fig. 1). Their structural features have been comprehensively described

Several forms of invariant chains can be found intracellularly associated with class 11 proteins. Two forms, 1131 and lidl span the membrane and expose the $\mathrm{N}$ terminus on the cytoplasmic side. They differ by an additional segment of 64 residues. which is found in li4l but not in 13., due to altemative splic ing ${ }^{10}$. This segment exhibits a high degree of similarity to a motif found in thyroglobulin (TgR element) and entactin, a protein found in the basement membrane". The cytoplasmic segment contains a cysteine residue which becomes fatty acylated during transport ${ }^{12}$. Ii chains are glycosylated at asparagine residues and a form of $\mathrm{I}$ also exists that contains a serine-linked chondroitin sulfate glycan side chain close to the C terminus ${ }^{1 / 3}$.

\section{Association of invariant chains with class II molecules and their intracellular transport}

Class II proteins and li associate very soon after their synthesis in the endoplasmic reticulum ${ }^{14}$. The oligomeric class II-Ii complex moves through the Golgi apparatus where the proteins become terminally glycosylated. Ii dissociates from class II polypeptides in a post-Golgi compartment where some of the Ii chains are proteolytically processed to peptides of 21 and $23 \mathrm{kDa}^{15}$. Processing and release of class II proteins from li can be inhibited by the protease inhibitor leupeptin. As chloroquine and $\mathrm{NH}_{4} \mathrm{Cl}$ also interfere with dissociation of class 11 molecules from Ii it has been suggested that it is an endosomal compartment in which dissociation occurs. This view is further supported by the finding that endocytosed transferrin receptor-ferritinneuraminidase conjugates meet $\mathrm{Ii}$ and class II polypeptides in an early endosomal compartment ${ }^{16}$. That $\mathrm{Ii}$ is also present in a late endosomal or a prelysosomal compartment is suggested by the proteolytic digestion of $\mathrm{Ii}$, and by its co-localization with mannose 
lumen

\section{$\mathrm{COOH}$}

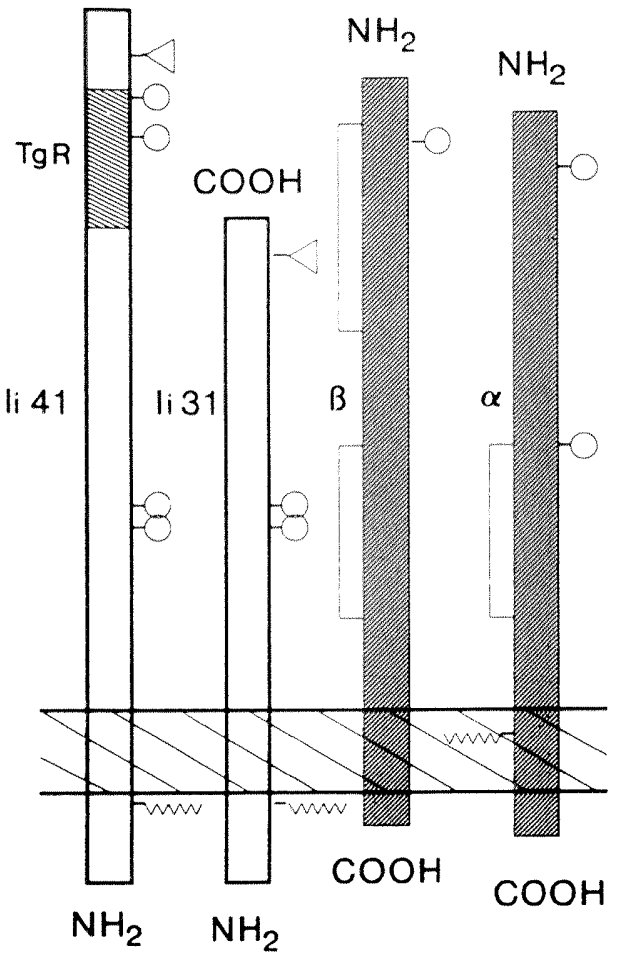

cytoplasm

Fig. 1. Schematic diagram of invariant chain (Ii) and class II polypeptides. The polypeptides IitI and Ii3I are encoded by the invariant chain gene and produced by differential splicing of a common preRNA. The thyroglobulin repeat element ( $T g R)$ in li4l is similar to a motif which is repeated ten times in thyroglobulin and is also found in entactin. The invariant chains Ii4I and Ii3I are type II membrane proteins which expose their $N$ terminus in the cytoplasm. Ii3I and Ii4l bear a cytoplasmic cysteine which is palmitylated ( $\neg \mathrm{w})$. Ii3l binds two, and Ii4l four, $\mathrm{N}$-linked glycan side chains $(-\bigcirc)$. Both Ii chains contain at their C-terminal end a glycosaminoglycan side chain $(-)$ ). The class II $\alpha$ and $\beta$ polypeptides are polymorphic type $I$ membrane proteins. The $\alpha$-chain has one intramolecular $S-S$ bond ( $\square$ ) and two $\mathrm{N}$-linked carbohydrates. The $\beta$-chain has two intramolecular $S-S$ bonds and one $\mathrm{N}$-linked carbohydrate. Only the $\alpha$-chain has a cysteine (which is fatty acylated) in the membrane-spanning region.

6-phosphate receptor (M6PR) (Lipp etal., unpublished).

Class II-Ii complexes are transported to an endosomal compartment where they dissociate and class II molecules move on to the cell surface, Ii remaining largely at an intracellular site. Transport of class II polypeptides to the endosomal compartment appears to be mediated by the Ii chain as, even in the absence of class II proteins, Ii can be found largely in this compartment (Lipp, unpublished). The path taken by class II polypeptide alone may be quite different from that of the class II-Ii complex (Fig. 2). Class
II polypeptides expressed in the absence of Ii are unlikely to enter an endosomal compartment. They probably move to the cell surface via the constitutive pathway taken by most secretory and resident cell surface proteins. Nothing is known about the structural elements involved in sorting of Ii chains to endosomal compartments. Ii chains share the membrane topology (type II membrane protein) with the transferrin receptor, a molecule which is endocytosed. For transferrin receptor (TR), it has been shown that the cytoplasmic tail is essential for endocytosis $^{17}$. A six amino acid motif in the cytoplasmic tail of li differs from that of the TR only in a replacement of the two glutamic acid residues by lysine $^{18}$. It is also conceivable that fatty acylation of the cytoplasmic tail or the chondroitin sulfate glycan side chains ${ }^{13}$ play a role in the sorting process.

Binding of Ii to class II polypeptides involves the extracytoplasmic domains of these molecules. Protease K cleavage of the cytoplasmic domains of $\mathrm{Ii}$ and class II chains did not influence the association ${ }^{14}$. Moreover an $18 \mathrm{kDa}$ extracytoplasmic fragment of $\mathrm{Ii}$ still associates with class II chains ${ }^{20}$.

\section{Co-expression and co-regulation of class II and invariant chains}

Class II $\alpha$ - and $\beta$-chains are encoded by genes of the major histocompatibility complex (found on mouse chromosome 17). The invariant chains are encoded by a gene located on chromosome 18. The pattern of expression of the invariant chain mirrors that of class II polypeptides, but Ii is also expressed in some other cell types which have no class $\mathrm{II}^{21}$. Class II proteins are expressed on B lymphocytes, macrophages, dendritic and some epithelial and endothelial cells. Some tissues require physiological signals such as interferon- $\gamma$ (IFN $\gamma)$ to induce class II or class II-Ii expression. The nonconstitutive expression of class II and Ii in non-immune cells could suggest that after invasion by infectious material a local activation of class II-Ii expression allows antigen processing/presentation to immigrating $\mathrm{T}$ lymphocytes and thereby elicits an immune response to the antigen.

Aberrant class II and invariant chain expression frequently coincides with an 'abnormal' situation such as inflammation during autoimmune disease. Hence the restriction of class II and invariant chain expression in the unstimulated state could prevent dys- function in the immune system, such as self destruction by autoimmunity.

\section{A role of invariant chains in antigen processing}

Since the class II-associated invariant chain was discovered ten years ago $^{22}$ several findings such as its coordinate expression with class II polypeptides and their co-regulation by IFN $\gamma^{23}$ and other cytokines, have been described. These and other observations raised the possibility of a functional role of the invariant chain in the immune response. Experimental evidence for such a role was obtained recently ${ }^{24}$. It was found that some class II transfected fibroblasts can present antigen only if the antigen is provided in fragmented form ${ }^{24,25}$. These cells have a deficiency to process/present intact antigen. However, if class II transfected fibroblast cells are also transfected with the invariant chain gene, these fibroblasts efficiently stimulate $\mathrm{T}$ cells ${ }^{24}$. Efficient stimulation of $T$ cells also occurs when only one of the two forms (Ii31 or Ii41) is expressed in these fibroblasts ${ }^{24}$. Stimulation occurs with both fragmented and intact antigen. Presentation of intact antigen, but not of fragmented antigen, was totally abrogated by treatment of fibroblasts with chloroquine. These experiments demonstrate that in these cells $\mathrm{Ii}$ is required for appropriate processing of antigen or for binding of processed antigen to class II protein. Consideration of the intracellular transport and location of class II proteins and invariant chain favours the idea that invariant chain is required for transport of class II proteins to a compartment containing processed antigen. This might be the same compartment in which class II proteins and Ii dissociate. Class II protein loaded with peptide then moves to the cell surface, whereas Ii is largely retained at an intracellular site. Several lines of evidence (see above) suggest that the compartment in which class II protein and Ii dissociate, and in which processed antigen is present, is a late endosome or a prelysosome. Interference of chloroquine with processing/presentation of intact antigen supports this notion. Chloroquine inhibits dissociation of class II and Ii and further transport of the complex ${ }^{26}$.

In the context of the posttranslational modifications of the invariant chains it is interesting to note that blocking fatty acylation with cerule$\operatorname{nin}^{27}$ as well as blocking proteoglycan formation with $p$-nitrophenylxyloside 


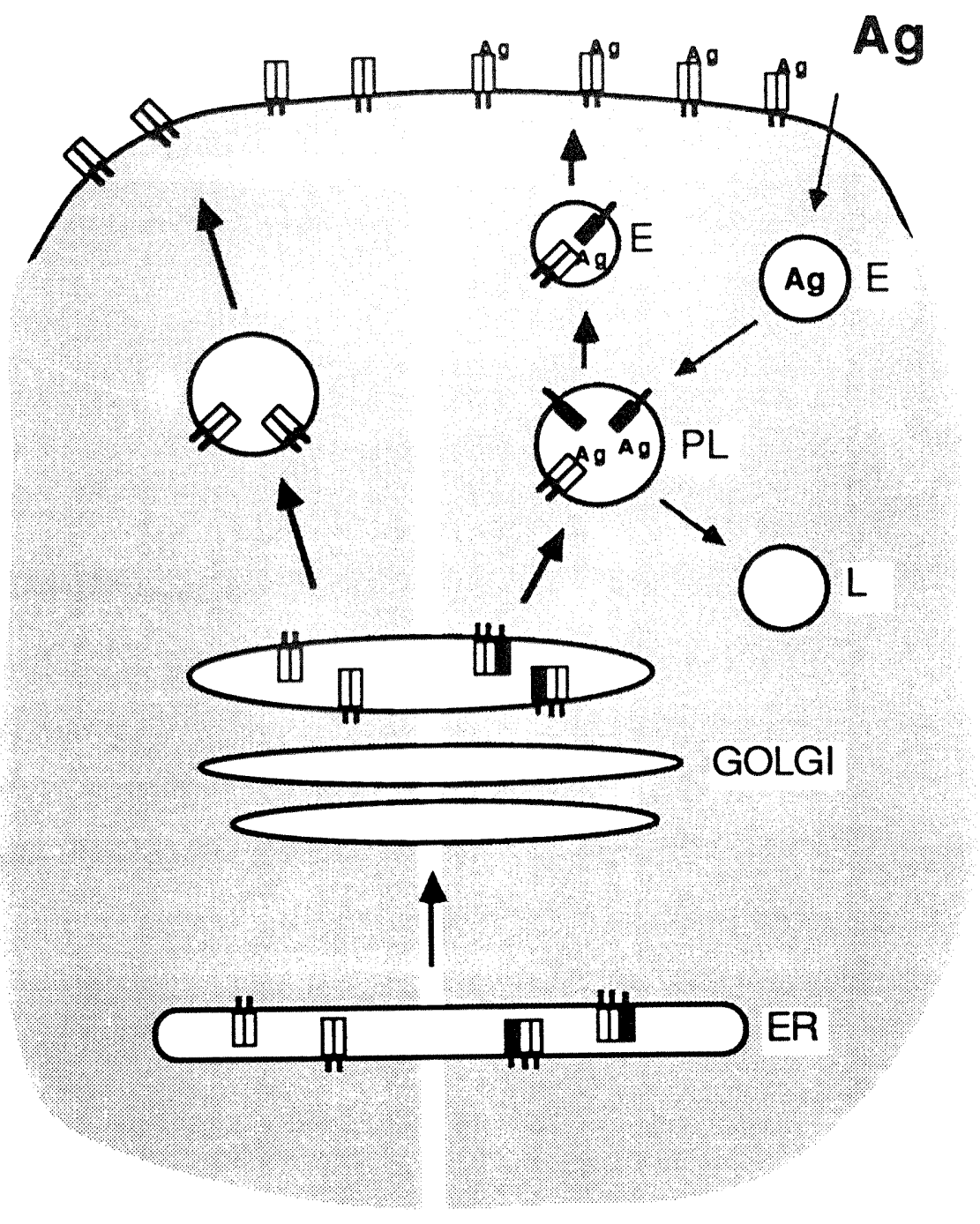

Fig. 2. Diagrammatic representation of postulated intracellular pathways taken by class II proteins and class II-Ii protein complexes. Open symbols indicate $\alpha$ - and $\beta$-chains of class II proteins; filled symbols represent Ii chains. Class II proteins and Ii associate in the membrane of the endoplasmic reticulum (ER) (right hand side), thereby preventing binding of peptide antigens to class II proteins. The oligomeric complex moves via the Golgi complex to a compartment where class II proteins dissociate from Ii chains. This compartment is characterized by a low pH and limited proteolytic activity. Several lines of evidence suggest that this compartment is a late endosome or a prelysosome (PL). Antigen ( $\mathrm{Ag}$ ) taken up by the cell (endocytosis) is partially degraded (processed) in this compartment before it is completely digested in lysosomes $(L)$. Class II proteins, after dissociation from $I i$, are expected to associate with processed antigen in the PL compartment and are then transported to the cell surface. Here they can present antigen to $T$ cells. Ii chains remain largely in intracellular compartments, PL and endosomes (E). In cells lacking Ii the class II proteins also assemble in the ER and are then transported via the Golgi complex to the cell surface (left hand side). The transport pathway taken to the cell surface might however be distinct from that taken by the class II-Ii complex. As class II proteins themselves do not have a sorting signal for an endosomal compartment, they are likely to move to the cell surface by the constitutive path way taken also by most of the secretory and resident plasma membrane proteins. In this case the class II proteins do not enter an endosomal or prelysosomal compartment during their transport to the cell surface and hence cannot take up processed antigen.

also inhibits antigen processing/ presentation by $\mathrm{APCs}^{28}$.

The Ii chains might also serve other roles in antigen processing apart from the transport function that we have discussed in this review. It has been suggested that a function of the invariant chain is to shield the peptide-binding site of newly synthesized class II until it reaches intracellular compartments where antigen is processed. Hence the binding site of the invariant chain to class II could be crucial for antigen processing. Several domains of the murine and the human Ii chain fulfill criteria to form amphipathic helixes which could be the site of interaction of Ii with the peptide binding groove of class II polypeptides ${ }^{29}$.

\section{Conclusions}

The experimental evidence suggests a functional role of the invariant chain in antigen processing/presentation. The intracellular compartment where antigen processing occurs is not yet known. If the invariant chains are involved in intracellular sorting of class II polypeptides, the site of dissociation could be of crucial importance for binding to processed antigen. Newly synthesized class II-Ii polypeptides could enter processing compartments directly after leaving the Golgi. The intracellular association of class II and li could have further significance. The association with Ii could protect the peptide-binding groove of class II polypeptides until peptides from the endocytic processing pathway are available. Unmasking of the peptide-binding domain at this stage may strongly increase the efficiency of antigenic peptide binding because there is no competition with previously bound peptide. This could explain how foreign peptides are presented but are not in competition with the large excess of self peptides.

\section{Acknowledgements}

This work was supported by grants from the Deutsche Forschungsgemeinschaft, Ko810/2-3 and Do 199/4-3. We thank Dr B. Arnold for critical comments on the manuscript and U. Edenharder for preparation of the figures.

\section{References}

1 Townsend, A. R. M., Gotch, F. M. and Davey, J. (1985) Cell 42, 457-467

2 Babbit, B. P., Allen, P. M., Matsueda, G., Haber, E. and Unanue, E. R. (1985) Nature $317,359-361$

3 Shimonkevitz, R., Kappler, J., Marrack, P. and Grey, H. (1983) J. Exp. Med. 158, 303316

4 Babbit, B. P., Matsueda, G., Haber, E., Unanue, E. R. and Allen, P. M. (1986) Proc. Natl Acad. Sci. USA 83, 4509-4513

5 Watts, T. H., Gaub, H. E. and McConnel, H. M. (1986) Nature 320, 179-181

6 Allen, P. M. (1987) Immunol. Today 8, 270273

7 Long, E. O. and Jacobson, S. (1989) Immunol. Today 10, 45-48

8 Diment, S., Leech, M. S. and Stahl, P. D. (1988) J. Biol. Chem. 263, 6901-6907

9 Kaufman, J. F., Auffray, C., Korman, A. J., Shackelford, D. A. and Strominger, J. (1984) Cell 36, 1-13

10 Koch, N., Lauer, W., Habicht, J. and Dobberstein, B. (1987) EMBOJ. 6, 1677-1683

11 Durkin, M.E., Chakravarti,S., Bartos, B. B., Liu, S-H., Friedman, R. L. and Chung, A. E. (1988) J. Cell. Biol. 107, 2749-2756

12 Koch, N. and Hämmerling, G. J. (1985) Biochemistry 24, 6185-6190

13 Miller, J., Hatch, J. A., Simonis, S. and Cullen, S. E. (1988) Proc. Natl Acad. Sci. USA 85, 1359-1363

14 Kvist, S., Wiman, K., Claesson, L., Peterson, P. A. and Dobberstein, B. (1982) Cell 29, $61-69$ 
15 Blum, J. S. and Cresswell, P. (1988) Proc. Natl Acad. Sci. USA 85, 3975-3979

16 Cresswell, P. (1985) Proc. Natl Acad. Sci. USA $82,8188-8192$

17 Rothenberger, S., Iacopetta, B. J and Kühn, L. C. (1987) Cell 49, 423-431

18 Koch, N. (1988) Biochemistry 27,4097-4102

19 Sant, A. J., Cullen, S. E., Giacoletto, K. S and Schwartz, B. D. (1985) J. Exp. Med. 162 1916-1934

20 Marks, M. S. and Cresswell, P. (1986) J Immunol. 136, 2519-2525
21 Momburg, F., Koch, N., Möller, P., Molden hauer, G., Butcher, G. W. and Hämmerling, G. J. (1986) J. Immunol. 136, 940-948

22 Jones, P. P., Murphy, D. B., Hewgill, D. and McDevitt, H. O. (1979) Immunochemistry $16,51-60$

23 Koch. N., Wong, G. H. W. and Schrader, J. W. (1986) J. Immunol. 132, 1361-1369

24 Stockinger, B., Pessara, U., Lin, R. H. Habicht, J, and Koch. N. (1989) Cell 56. $683-689$

25 Shastri, N., Malissen, B. and Hood, L. (1985)
Proc. Natl Acad. Sci. USA 82, 5885-5889

26 Nowell, J. and Quaranta, V. (1985) J. Exp. Med. $162,1371-1376$

27 Falo, L. D.. Benacerraf, B., Rothstein, L. and Rock, K. L. (1987) J. Immunol. 139, 3918-3923

28 Rosamond, S., Brown, L., Gomez, C. Braciale, T. J. and Schwartz, B. D. (1987) J. Immunol. 139, 1946-1951

29 Elliott, W. L. Stille, C. J., Thomas, L. J. and Humphreys, R. E. (1987) J. Immunol. 138, 2949-2952

\title{
Reflections on Biochemistry
}

\section{On the tracks of our scientific forbears}

\author{
Fritz Schlenk
}

Delving into the history of biochemistry is an activity in which scientists in the prime of their productivity rarely engage. No issue can be taken with this, because the investigator will hardly ever derive information that can be applied to his current work. Active biochemists have the healthy attitude of looking forward rather than backward, and to learn from their contemporaries and competitors rather than from activities of the past. As one enters a less active phase of life, however, much pleasure and amusement, in addition to interesting information about the past, can be derived from roaming through early biochemical publications. The truly great accomplishments of early biochemistry and its cognate sciences have been covered in several monographs ${ }^{1}$. Here we shall look at some more personal impressions of our predecessors and the circumstances surrounding them. Only a few glimpses of the past can be given.

\section{Treasures in the library}

What one finds in the library varies a good deal; the major historical treatises often are so concise that much interesting detail is lost. Far more personal impressions are derived from autobiographical accounts, such as the prefatory chapters published since 1953 in the Annual Reviews of Biochemistry, in

F. Schlenk is at the University of Illinois at Chicago, Department of Biological Sciences, Box 4348, Chicago, IL 60680-066, USA. each edition of which one of the important biochemists of advanced age reminisces about his past. It is, as Albert von Szent-Györgyi remarks at the outset of his tale, as if he were taking a candle and going out himself to his funeral ${ }^{2}$.

These autobiographical chapters are usually written with great modesty. Very different are the 'Festschriften' of the past, sometimes comprising an entire volume of a professional journal, paying homage to a prominent scientist at his 60 th, 70th, or later anniversary. A prefatory chapter, written by a close associate, stresses the wonderful traits of the person honored, followed by contributions of his former students who relish to be recognized as scientific offspring of the master. Memorial volumes and obituaries resemble these affectionate reports in many ways. However, they are reliable sources of the biographical data, the professional stations, and often they contain a list of the most important publications of the deceased. In the past, these discourses used to be very long, often including non-professional accomplishments. For example, in Eduard Buchner's obituary ${ }^{3}$ his prowess as an alpinist is documented by a list of his most difficult ascents and 'firsts'.

\section{Meager professional prospects, belated recognition}

The early leaders of biochemistry rarely had set their goals at the outset of their studies. Biochemical careers were not obvious during the first part of the past century, and departments and chairs of biochemistry did not yet exist. Most of the early biochemists started as physicians or chemists, and often they stayed with their initial specialty while making important contributions also to biochemistry. The interplay between microbiology and biochemistry came much later. Pasteur showed the interdependence of these fields, but it took several decades more before close contact between these two disciplines became widespread.

In Germany, one of the first departments of Physiological Chemistry was created for Felix Hoppe-Seyler in 1872, at the University of Strasbourg. For chemists, the situation was not much better. For example, the preamble to the foundation of the German Chemical Society in 1867 states as one of the encouraging reasons that the University of Berlin now, for the first time, had a special unit for the training of chemistry students ${ }^{4}$. None of the modern comforts and safety features existed in the laboratories. From early letters of Liebig we learn that during his post-doctoral stay with J. L. GayLussac in Paris (1822-1824) he had to suffer extreme discomfort during the winter in a primitive unheated laboratory. A picture of Liebig's first laboratory at the University of Giessen in 1842 makes one wonder how the staff in tails or morning coat, some wearing top hats, could do experiments in the highly crammed and primitive quarters ${ }^{5}$ (Fig. 1).

The development and rapid growth of the chemical industries and the recognition by the medical professions that chemistry could aid the 'health sciences' brought on the development of special curricula in all universities.

The financial compensation of chemists and biochemists in academic positions was moderate; only at advanced 